\title{
Integration of haptics and vision in human multisensory grasping
}

Ivan Camponogara and Robert Volcic

Department of Psychology, New York University Abu Dhabi, Abu Dhabi, United Arab Emirates

\section{Author names and affiliations:}

Dr. Ivan Camponogara

Department of Psychology, New York University Abu Dhabi

PO Box 129188, Abu Dhabi, United Arab Emirates

E-mail: ivan.camponogara@nyu.edu

Dr. Robert Volcic

Department of Psychology, New York University Abu Dhabi

PO Box 129188, Abu Dhabi, United Arab Emirates

E-mail: robert.volcic@nyu.edu

Corresponding author email address: ivan.camponogara@nyu.edu 


\begin{abstract}
:
Grasping actions are directed not only toward objects we see but also toward objects we both see and touch (multisensory grasping). In this latter case, the integration of visual and haptic inputs improves movement performance compared to each sense alone. This performance advantage could be due to the integration of all the redundant positional and size cues or to the integration of only a subset of these cues. Here we selectively provided specific cues to tease apart how these different sensory sources contribute to visuo-haptic multisensory grasping. We demonstrate that the availability of the haptic positional cue together with the visual cues is sufficient to achieve the same grasping performance as when all cues are available. These findings provide strong evidence that the human sensorimotor system relies on non-visual sensory inputs and open new perspectives on their role in supporting vision during both development and adulthood.
\end{abstract}

Keywords: Multisensory integration, vision, haptics, proprioception, touch, grasping 


\section{Introduction}

The planning and execution of a successful reach-to-grasp movement relies on the translation of visual inputs into motor commands that are involved in the control of the hand (Castiello, 2005; Janssen \& Scherberger, 2015; Jeannerod, Arbib, Rizzolatti, \& Sakata, 1995). However, actions in everyday life are not only directed toward objects we see but also toward objects we feel with our hands. For instance, even without looking at it, we can easily reach and grasp the cap of a pen while holding the pen with the other hand. Thus, the afferent haptic inputs (proprioception and touch) from the hand in contact with the object are sufficient to estimate the object's position and its size and successfully guide the action of the opposite hand. However, movements toward haptically sensed objects are usually slower and show a wider grip aperture compared to movements directed toward visually detected objects (Camponogara \& Volcic, 2019b; Pettypiece, Culham, \& Goodale, 2009; Pettypiece, Goodale, \& Culham, 2010). Importantly, the simultaneous availability of vision and haptics leads to faster movements with narrower grip apertures compared to when objects are only visually or haptically sensed (Camponogara \& Volcic, 2019a, 2019b). Thus, haptics can effectively integrate with vision to plan and execute grasping actions, but how does each sense contribute to this multisensory-motor transformation is still poorly understood.

Even though vision and haptics provide redundant cues about the position and the size of an object, the two sensory systems acquire and process these cues in fundamentally different ways. In the visual domain, the estimated size of an object is tightly linked to its estimated position in depth. In essence, determining an object's size requires the scaling of its retinal projections according to its distance from the observer (Brenner \& van Damme, 1999; Epstein, Park, \& Casey, 1961; van Damme \& Brenner, 1997; Volcic \& Domini, 2018; Volcic, Fantoni, Caudek, Assad, \& Domini, 2013). Instead, in the haptic domain, the estimated size and position of the object are independent. The object size is provided by the proprioceptive and tactile inputs from the digits enclosing the object (Berryman, Yau, \& Hsiao, 2006; Durlach et al., 1989; Gaydos, 1958; Langfeld, 1917), whereas the object position is provided by the proprioceptive inputs from the muscles, joints, and skin of the flexed arm (Proske \& Gandevia, 2012). Hence, if we assume that the perceived size and position of the objects are used to control grasping movements, there are multiple ways in which visual and haptic cues could be integrated in multisensory grasping. For instance, multisensory grasping could be based on the integration of all visual and haptic position and size cues or only on a subset of these. We captured the different degrees of integration with three models: the full integration, the size integration and the position integration 
model (Figure 1).

Full integration

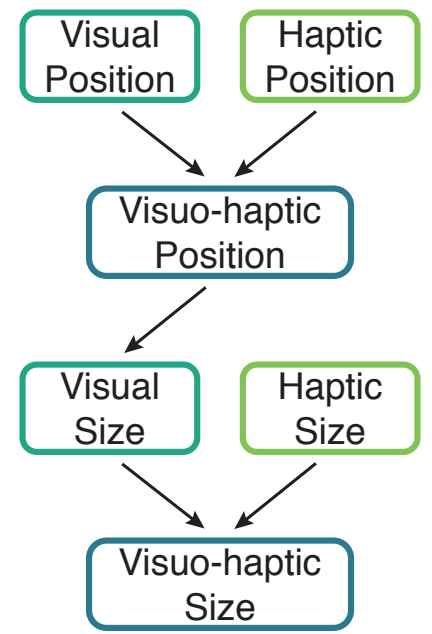

Size integration

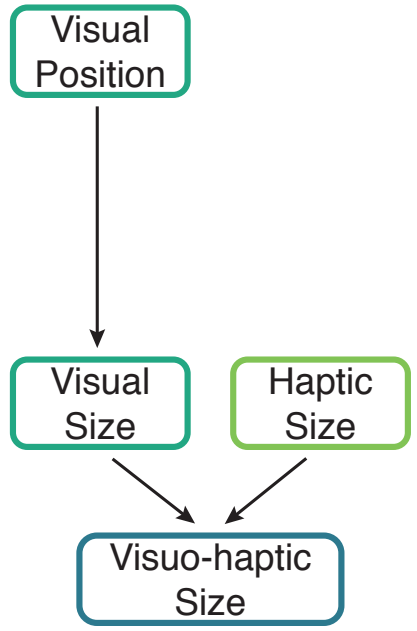

Position integration
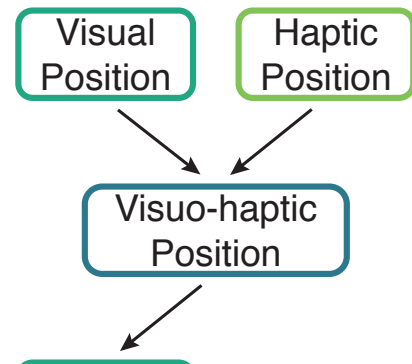

Visual

Size

Figure 1: Integration of visual and haptic cues. Left panel, Full integration model: visual and haptic position estimates are merged into a visuo-haptic position estimate. The visual size estimate, which is based on the multisensory position estimate is then integrated with the haptic size estimate. Middle panel, Size integration model: visual object size is mainly based on the visual position estimate. The visual size estimate is then integrated with the haptic size estimate. Right panel, Position integration model: visual and haptic position estimates are integrated into a visuo-haptic position estimate which supports the visual object size estimate.

A full integration model posits that both visual and haptic cues about the object size and its position are all merged (Figure 1, left panel): the integration of visual and haptic position estimates promotes a better visual size estimate which is then integrated with the haptic size estimate. The enhanced performance in visuo-haptic multisensory grasping would thus stem from the availability of multisensory estimates of both position and size. An alternative model, the size integration model (Figure 1, middle panel), minimizes the role of the haptic position cue and its output is thereby mainly influenced by the integration of haptic and visual size estimates. The joint availability of visual and haptic size would be thus sufficient to improve performance in visuo-haptic grasping. This model resembles the models commonly used to describe visuo-haptic size perception (Ernst \& Banks, 2002; Ernst \& Bülthoff, 2004; Gepshtein \& Banks, 2003; Gepshtein, Burge, Ernst, \& Banks, 2005). Another model, the position integration model (Figure 1, right panel), is instead focused on the role of haptics as an auxiliary position information cue (Battaglia et al., 2010; Carey \& Allan, 1996; Chen, Sperandio, \& Goodale, 2018; Sperandio, Kaderali, Chouinard, Frey, \& Goodale, 2013, but see Brenner, van Damme, \& Smeets, 1997). The output of this model is thus mainly determined by the integration of haptic and visual position estimates with the haptic size cue 
playing only a marginal role. In this case, the haptic position estimate would improve the visual position estimate which would lead to a better visual size estimate and, in turn, boost visuo-haptic multisensory grasping.

To determine the contribution of haptic and visual cues in multisensory grasping we have characterized grasping performance in two unisensory and two multisensory conditions (Figure 2a). In all conditions the to-be-grasped objects varied in size along the depth dimension and were positioned at different egocentric distances. In the haptic condition $(\mathrm{H})$, vision was prevented, and grasping relied on the haptic size and position cues provided by the left hand holding the object. In the visual condition (V), grasping was based on the visually sensed location and size of the object. In the first multisensory condition, the visuo-haptic condition $(\mathrm{VH})$, both visual and haptic cues about the object position and its size were available. In the second multisensory condition, the visuo-haptic position condition (VHP), participants held with the left hand a post which supported the object, instead of holding the object itself, while vision was fully available. Thus, haptics was informative only about the position of the object, but not about its size. In all these conditions, the grasping action was performed by the right hand.

Based on previous research (Camponogara \& Volcic, 2019a, 2019b), we expect the multisensory (VH) condition to exhibit faster grasping movements with smaller peak grip apertures than the $\mathrm{V}$ and $\mathrm{H}$ unisensory conditions. Whereas all three integration models predict improved grasping performance compared to when objects are only visually or haptically sensed, the comparison between the two multisensory conditions (VH vs. VHP) is instead crucial to distinguish among the full integration, size integration and position integration models. Both the full integration and the size integration models rely, in part, on the availability of the haptic size cue to achieve the multisensory advantage. Thus, if the haptic size cue is absent, both models predict a worse grasping performance in VHP (in which no haptic size cue is provided) than in VH. Grasping movements should be as slow and with peak grip apertures as large as in the $\mathrm{V}$ condition according to the size integration model and somewhere in-between the $\mathrm{VH}$ and the V conditions according to the full integration model. On the other hand, the position integration model depends mainly on the availability of the haptic position cue. Hence, if only the haptic position cue is sufficient, this model predicts grasping movements in VHP to be as fast and with the same grip aperture as in $\mathrm{VH}$. 


\section{Materials and methods}

No part of the study procedures or study analyses were pre-registered prior to the research being conducted. We report how we determined our sample size, all data exclusions, all inclusion/exclusion criteria, whether inclusion/exclusion criteria were established prior to data analysis, all manipulations, and all measures in the study.

\section{$2.1 \quad$ Participants}

Decisions about the sample size were taken prior to data collection and were based on a recent similar study (Camponogara \& Volcic, 2019b) in which the same behavioral paradigm was used. However, we have increased the number of trials per participant to maximize power. Inclusion/exclusion criteria for participants were established prior to the experiment, including normal or corrected-to-normal vision, right-handedness (self-reported), above 18 years of age, and no known history of neurological disorders. No participant was excluded. Twenty students from the New York University Abu Dhabi took part in this study (10 males, age $19.4 \pm 0.9)$. All participants were provided with a subsistence allowance. The experiment was undertaken with the understanding and written informed consent of each participant and experimental procedures were approved by the Institutional Review Board of New York University Abu Dhabi.

\section{$2.2 \quad$ Apparatus}

In the $\mathrm{H}, \mathrm{V}$ and $\mathrm{VH}$ conditions, the stimuli were five 3D-printed rectangular cuboids with a depth of 30 , 40, 50, 60, $70 \mathrm{~mm}$, all the same height (120 mm) and width (25 mm) (Figure 2b). In the VHP condition, the stimuli were five $60 \mathrm{~mm}$ high rectangular cuboids supported by a $60 \mathrm{~mm}$ high post (Figure 2c). While the upper part of these objects varied in depth as in the first set of objects, the depth of the post was kept constant $(10 \mathrm{~mm})$ and thus haptics was non-informative about the depth of the to-be-grasped object. In addition, we have manipulated the position of the objects by placing them at three different egocentric distances in front of the participants: at $150 \mathrm{~mm}, 250 \mathrm{~mm}$ or $350 \mathrm{~mm}$ in the sagittal direction (depth) at table height. Two $5 \mathrm{~mm}$ high rubber bumps with a diameter of $9 \mathrm{~mm}$ were attached just in front of the participants, $300 \mathrm{~mm}$ to the left and to the right. These bumps were marking the start positions for the left and right hands (Figure 2b).

A pair of occlusion goggles (Red Scientific, Salt Lake City, UT, USA) controlled by a custom Matlab 
program was used to prevent vision of the workspace in the haptic condition and between trials. A pure tone of $1000 \mathrm{~Hz}$ and $100 \mathrm{~ms}$ length was used to signal the start of the trial, while another tone of $600 \mathrm{~Hz}$ with the same duration was used to signal its end. Index, thumb and wrist movements were acquired on-line at $200 \mathrm{~Hz}$ with sub-millimeter resolution by using an Optotrak Certus system (Northern Digital Inc., Waterloo, Ontario, Canada) controlled by the MOTOM toolbox (Derzsi \& Volcic, 2018). The position of the tip of each digit was calculated during the calibration phase with respect to three infrared-emitting diodes attached on each distal phalanx (Nicolini, Fantoni, Mancuso, Volcic, \& Domini, 2014). An additional marker was attached on the wrist (styloid process of the radius).

\subsection{Procedure}

Participants sat comfortably in front of a table with their torso touching its edge. All the trials started with the participants' thumb and index digit of the right and left hand positioned on the respective start positions and the shutter goggles closed. Before each trial, one of the objects was positioned at one of the three positions. In the $\mathrm{H}$ condition, the experimenter signaled to the participants to hold the object with their left hand along its depth axis at its base (i.e., sense its size and position by means of tactile and proprioceptive inputs) while the shutter goggles remained closed. In the $\mathrm{V}$ condition, the goggles turned transparent to provide vision of the object, no haptic information was provided by the left hand. In the $\mathrm{VH}$ condition, the participants held the object with their left hand and then the goggles turned transparent. In the VHP condition, participants held the post on which the object was firmly placed with their left hand and then the goggles turned transparent. Unlike in VH, in which haptic inputs were informative about both the object size and its position, the haptic inputs in VHP were informative only about the object's position (Figure 2c).

After a variable period, the start tone was delivered and participants had to reach for and grasp the object along its depth axis. Movements were performed at a natural speed and no reaction time constrains were imposed. After 3 seconds the end sound was delivered, and, only in the H modality, the goggles were made transparent. Participants had to move their right and left hands back to the start positions and then the goggles turned opaque. Another object and position were then selected and the next trial was ready to start.

The order of conditions was randomized across participants using a Latin square design, while size and position configurations were randomized within each condition. We ran five repetitions for each combination of object size and position, which led to a total of 300 trials per participant (75 for each 

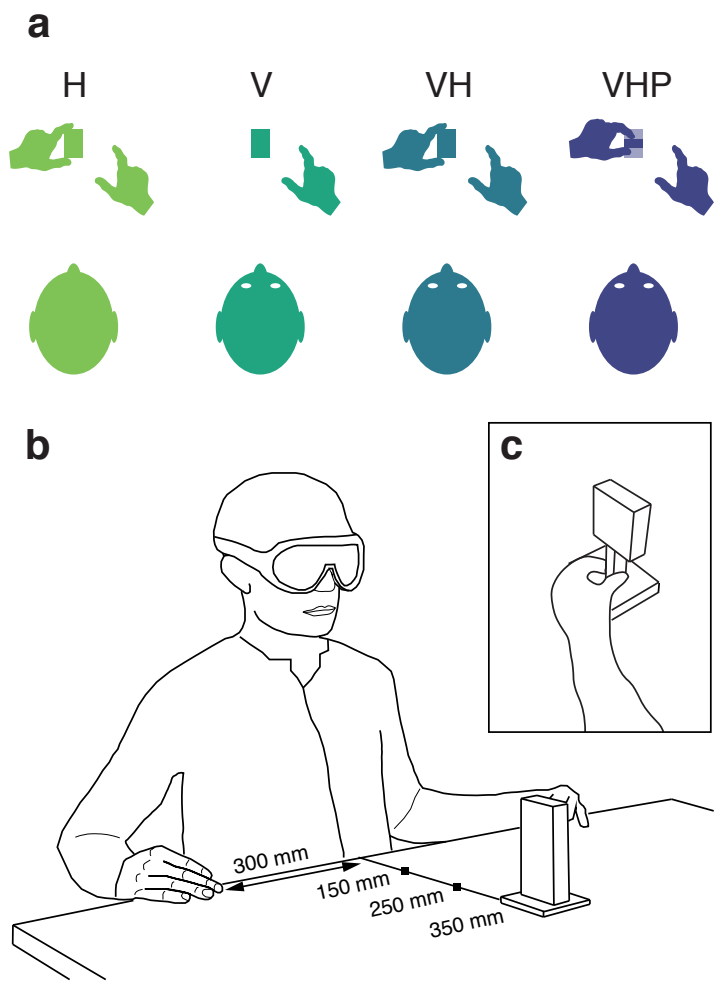

Figure 2: (a) Haptic (H), visual (V), visuo-haptic (VH), and visuo-haptic position (VHP) conditions. Grasping actions were always performed with the right hand. In H, VH and VHP the left hand was already holding the object or the post before the start of the grasping movement. (b) Experimental setup with the stimulus used in conditions $\mathrm{H}, \mathrm{V}$, and VH. (c) Left hand holding the post that supported the object in condition VHP.

condition). Before the experiment, a training session was performed in which ten trials were run in each condition to accustom the participants with the task.

\subsection{Data analysis}

Kinematic data were analyzed in $\mathrm{R}$ (R Core Team, 2019). The raw data were smoothed and differentiated with a third-order Savitzky-Golay filter with a window size of 21 points. These filtered data were then used to compute velocities and accelerations in three-dimensional space for each digit and the wrist. Movement onset was defined as the moment of the lowest, non-repeating wrist acceleration value prior to the continuously increasing wrist acceleration values (Camponogara \& Volcic, 2019b; Volcic \& Domini, 2016), while the end of the grasping movement was defined on the basis of the Multiple Sources of Information method (Schot, Brenner, \& Smeets, 2010). We used the criteria that the grip aperture is close to the size of the object, that the grip aperture is decreasing, that the second derivative of the grip 
aperture is positive, and that the velocities of the wrist, thumb and index finger are low. Moreover, the probability of a moment being the end of the movement decreased over time to capture the first instance in which the above criteria were met (Camponogara \& Volcic, 2019b; Volcic \& Domini, 2016). Trials in which the end of the movement was not captured correctly or in which the missing marker samples could not be reconstructed using interpolation were discarded from further analysis. The exclusion of these trials (90 trials, $1.5 \%$ in total) left us with 5910 trials for the final analysis.

We focused our analyses on two dependent variables: the peak grip aperture, defined as the maximum Euclidean distance between the thumb and the index finger, and, the peak velocity of the hand movement, defined as the highest wrist velocity along the movement. These two variables are indicative of the effect of object size and the effect of object position on the movement kinematics (Jeannerod, 1981).

We analyzed the data using Bayesian linear mixed-effects models, estimated using the brms package (Bürkner, 2017) which implements Bayesian multilevel models in R using the probabilistic programming language Stan (Carpenter et al., 2017). The models used to fit the peak grip aperture and peak velocity data included as fixed-effects (predictors) the categorical variable Condition ( $\mathrm{H}, \mathrm{V}, \mathrm{VH}, \mathrm{VHP})$ in combination with the continuous variables Size and Position. The continuous variables Size and Position were centered before being entered in the models, thus, the estimates of the Condition parameters $\left(\beta_{\text {Condition }}\right.$ intercepts) correspond to the average performance of each Condition. The estimates of the parameters Size ( $\beta_{\text {Size }}$ slope) and Position ( $\beta_{\text {Position }}$ slope) correspond instead to the change in the dependent variables as a function of the object size and its position (i.e., distance from the participant). All models included independent random (group-level) effects for subjects. Models were fitted considering weakly informative prior distributions for each parameter to provide information about their plausible scale. We used Gaussian priors for the Condition fixed-effect predictor (peak grip aperture $\beta_{\text {Condition }}$ : mean $=85$ and $\mathrm{sd}=40 ;$ peak velocity $\beta_{\text {Condition }}$ : mean $=950$ and $\left.\mathrm{sd}=500\right)$. For the Size and Position fixed-effect predictors we used Cauchy prior distributions centered at 0 with a scale parameter of 2.5. For the grouplevel standard deviation parameters and sigmas we used Student $t$-distribution priors (peak grip aperture all sd parameters and sigma: $d f=3$, scale $=16$; peak velocity sd Condition and sigma: $d f=3$, scale $=$ 166; peak velocity sd Size and sd Position: $d f=3$, scale $=5$ ). Finally, we set a prior over the correlation matrix that assumes that smaller correlations are slightly more likely than larger ones (LKJ prior set to $2)$.

For each model we ran four Markov chains simultaneously, each for 16,000 iterations (1,000 warm-up 
samples to tune the MCMC sampler) with the delta parameter set to 0.9 for a total of 60,000 postwarm-up samples. Chain convergence was assessed using the $\hat{R}$ statistic (all values equal to 1 ) and visual inspection of the chain traces. Additionally, predictive accuracy of the fitted models was estimated with leave-one-out cross-validation by using the Pareto Smoothed Importance Sampling. All Pareto k values were below 0.5 .

The posterior distributions we have obtained represent the probabilities of the parameters conditional on the priors, model and data, and, they represent our belief that the "true" parameter lies within some interval with a given probability. We summarize these posterior distributions by computing the medians and the $95 \%$ Highest Density Intervals (HDI). The 95\% HDI specifies the interval that includes with a $95 \%$ probability the true value of a specific parameter. To evaluate the differences between parameters of two conditions, we have simply subtracted the posterior distributions of $\beta_{\text {Condition }}, \beta_{\text {Size }}$ and $\beta_{\text {Position }}$ weights between specific conditions (H-V, VH-H, VH-V, VH-VHP). The resulting distributions are denoted as the credible difference distributions and are again summarized by computing the medians and the $95 \%$ HDIs.

For statistical inferences about model parameters $\left(\beta_{\text {Size }}\right.$ and $\left.\beta_{\text {Position }}\right)$ we assessed the overlap of the 95\% HDI with zero. A 95\% HDI that does not span zero indicates that the predictor has an effect on the dependent variable. For statistical inferences about the differences of the model parameters between conditions, we applied an analogous approach. A 95\% HDI of the credible difference distribution that does not span zero is taken as evidence that the model parameters in the two conditions differ from each other.

\section{Results}

We report the main results in two separate sections. In the first section, we describe and compare the performance in the $\mathrm{H}, \mathrm{V}$ and $\mathrm{VH}$ conditions to establish how unisensory and multisensory inputs affects grasping behavior. In the second section, we focus on the critical comparison between the VH and VHP conditions. This comparison allows us to establish whether the haptic size cue is crucial to improve multisensory grasping movements and will reveal which model (full integration, size integration or position integration) is the most plausible. 


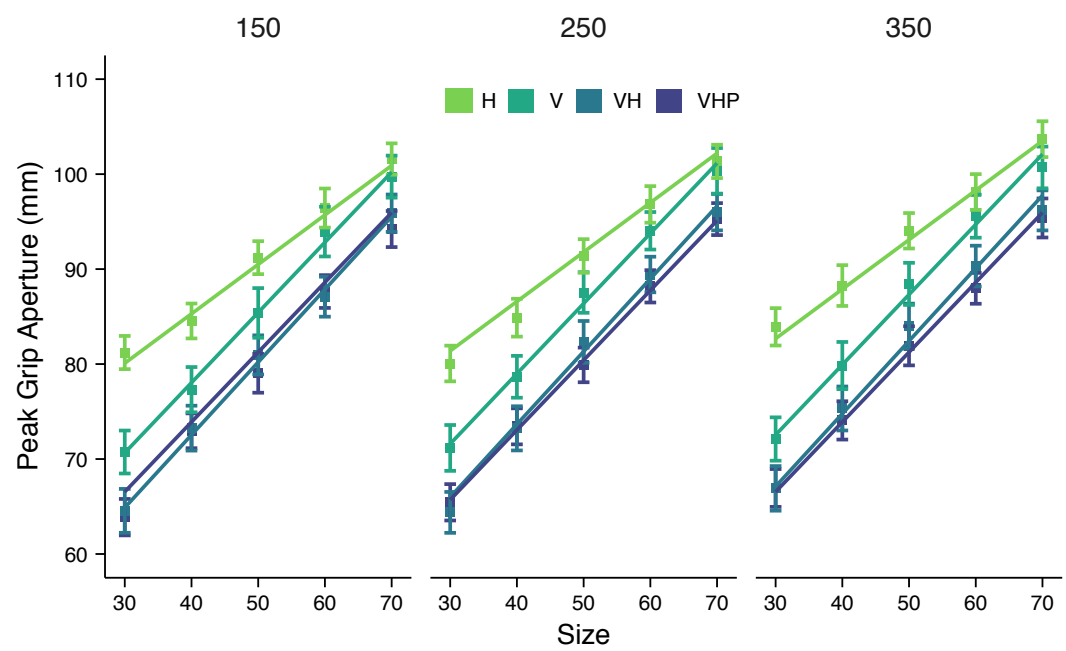

Figure 3: Average peak grip aperture as a function of size and position (separate panels) in the H, V, VH and VHP conditions. Error bars represent the standard error of the mean. Solid lines show the Bayesian mixed-effects model fits.

\subsection{Grasping with haptic, visual and visuo-haptic inputs}

The peak grip aperture was clearly affected not only by the size and position of the object, but, most importantly, also by the available sensory inputs (Figure 3). As can be also seen in Figure 4a, the average peak grip aperture was larger in $\mathrm{H}(91.8 \mathrm{~mm}, 95 \% \mathrm{HDI}=88.2,95.0)$ than in $\mathrm{V}(86.3 \mathrm{~mm}, 95 \% \mathrm{HDI}=$ $82.5,90.3)$ which, in turn, was larger than in VH $(81.2 \mathrm{~mm}, 95 \% \mathrm{HDI}=77.7,84.7)$. The comparisons between conditions (Figure 4d), showed that the peak grip aperture in $\mathrm{H}$ was credibly larger than in both $\mathrm{V}(\mathrm{H}-\mathrm{V}=5.4 \mathrm{~mm}, 95 \% \mathrm{HDI}=1.3,9.4)$ and $\mathrm{VH}(\mathrm{H}-\mathrm{VH}=10.5 \mathrm{~mm}, 95 \% \mathrm{HDI}=6.7,14.5)$. Moreover, the peak grip aperture in $\mathrm{V}$ was credibly larger than in $\mathrm{VH}(\mathrm{V}-\mathrm{VH}=5.1 \mathrm{~mm}, 95 \% \mathrm{HDI}=$ $2.1,8.0$ ). These peak grip aperture changes were very consistent across participants (Figure 5a, b, and c). The result that the peak grip aperture is smallest in $\mathrm{VH}$ replicates and extends previous findings (Camponogara \& Volcic, 2019a, 2019b), and supports the idea that the simultaneous availability of visual and haptic inputs leads to a substantial multisensory advantage (approximately $5 \mathrm{~mm}$ and $10 \mathrm{~mm}$ smaller average peak grip aperture compared to $\mathrm{V}$ and $\mathrm{H}$, respectively).

The peak grip aperture increased as a function of object size in all sensory conditions (Figure 3). However, the slope was shallower in $\mathrm{H}(0.52,95 \% \mathrm{HDI}=0.49,0.54)$ than in $\mathrm{V}(0.73,95 \% \mathrm{HDI}=0.71,0.76)$ and VH $(0.76,95 \%$ HDI $=0.73,0.80)$, as can be seen in Figure $4 \mathrm{~b}$. The comparisons between conditions (Figure 4e) showed that peak grip aperture increased credibly less as a function of object size in $\mathrm{H}$ than 

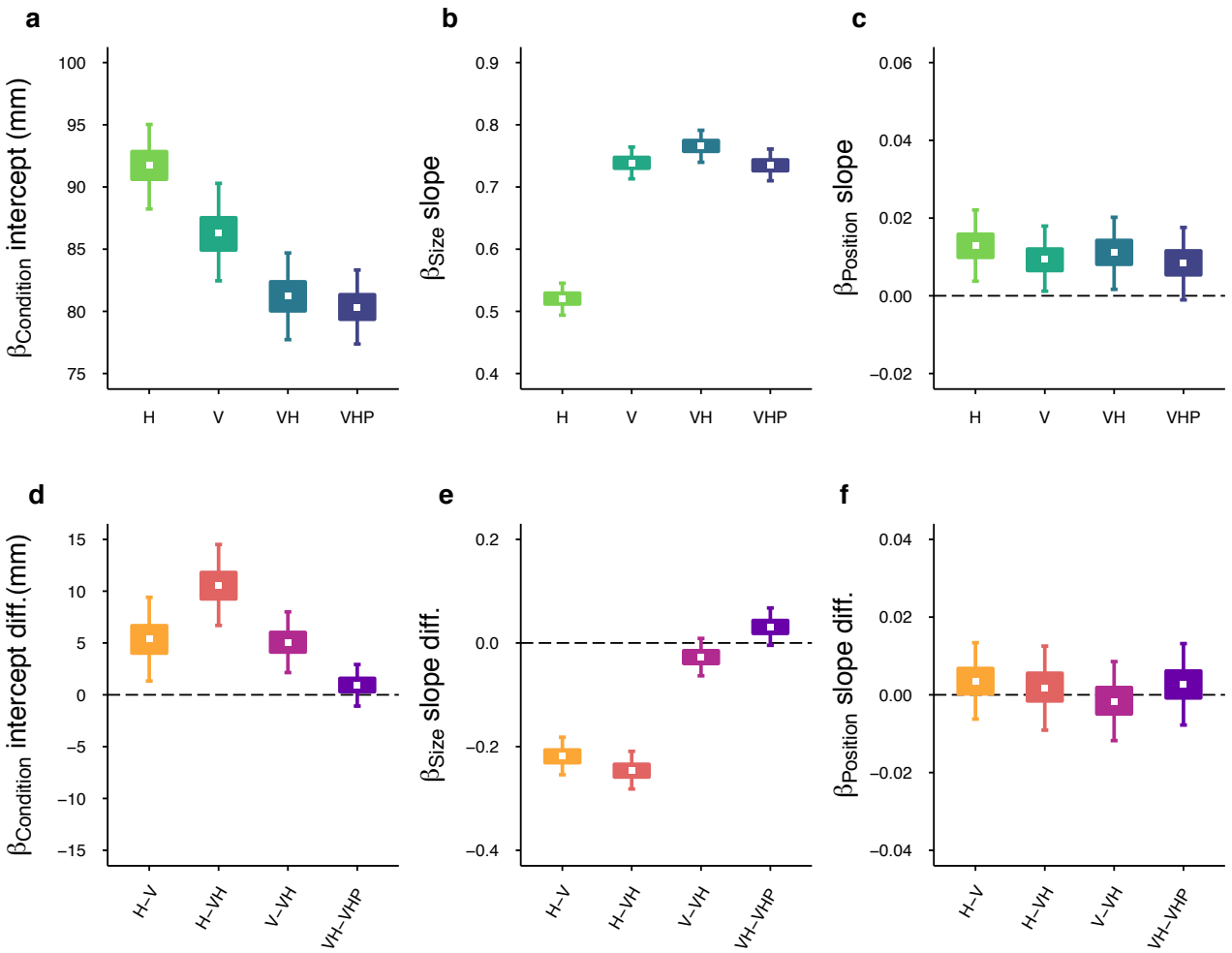

Figure 4: Peak grip aperture results. Top row: Posterior beta weights of the Bayesian linear mixed-effects regression model for the predictors Condition (a), Size (b) and Position (c). Bottom row: Credible difference distributions between conditions for the predictors Condition (d), Size (e) and Position (f). White dots represent the median, the boxes represent the 50\% HDIs, and the areas between whiskers represent the $95 \%$ HDIs of the distributions.

in both $\mathrm{V}(\mathrm{H}-\mathrm{V}=-0.21,95 \% \mathrm{HDI}=-0.25,-0.18)$ and $\mathrm{VH}(\mathrm{H}-\mathrm{VH}=-0.24,95 \% \mathrm{HDI}=-0.28$, -0.20). Instead, the changes in peak grip aperture as a function of object size were essentially identical in $\mathrm{V}$ and $\mathrm{VH}(\mathrm{V}-\mathrm{VH}=-0.02,95 \% \mathrm{HDI}=-0.06,0.01)$. This suggests that the peak grip aperture in $\mathrm{H}$ is less sensitive to changes in object size and, thus, the peak grip aperture modulation in multisensory grasping is mainly based on the visual size cue.

The peak grip aperture credibly increased also as a function of object position (Figure 4c), but only moderately $(\mathrm{H}=0.012,95 \% \mathrm{HDI}=0.003,0.022 ; \mathrm{V}=0.009,95 \% \mathrm{HDI}=0.001,0.018, \mathrm{VH}=0.011$, 95\% HDI $=0.001,0.02$ ). Most importantly, the comparisons between conditions (Figure 4f) showed that these peak grip aperture increases were indistinguishable between conditions $(\mathrm{H}-\mathrm{V}=0.003,95 \%$ $\mathrm{HDI}=-0.005,0.01 ; \mathrm{H}-\mathrm{VH}=0.001,95 \% \mathrm{HDI}=-0.008,0.01 ; \mathrm{V}-\mathrm{VH}=-0.001,95 \% \mathrm{HDI}=-0.01$, $0.007)$. 

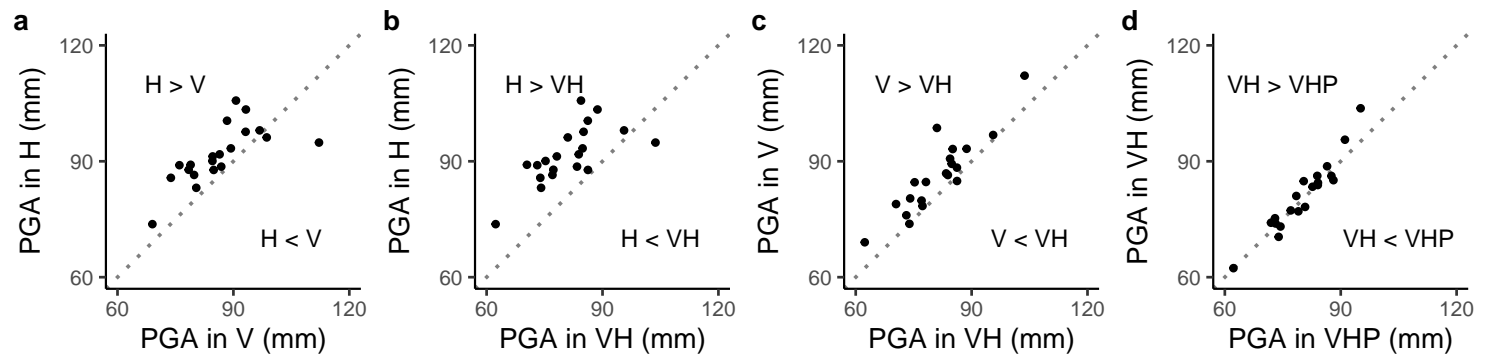

Figure 5: Scatterplots of paired observations. Each point represents the average peak grip aperture (PGA) of a single participant for a pair of conditions: (a) V and H, (b) VH and H, (c) VH and V, (d) VHP and VH. The diagonal reference line of no effect has slope 1 and intercept 0 . Points above the diagonal line indicate that the peak grip aperture of the condition represented on the ordinate axis is larger than the peak grip aperture represented on the abscissa.

150

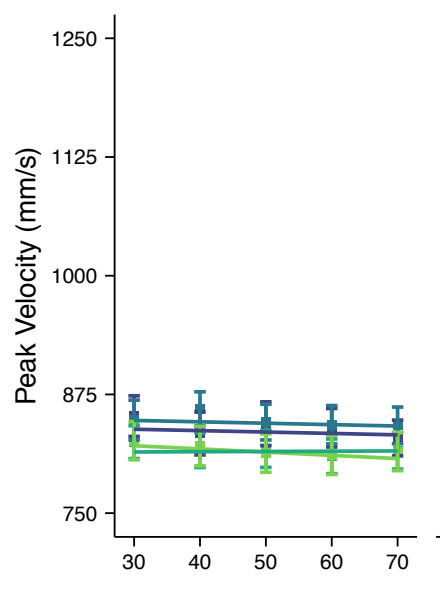

250

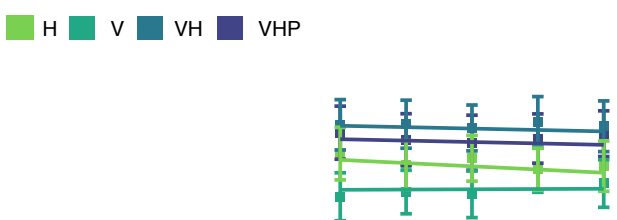

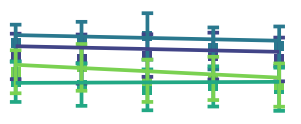

350

Figure 6: Average peak velocity as a function of size and position (separate panels) in the H, V, VH and VHP conditions. Error bars represent the standard error of the mean. Solid lines show the Bayesian mixed-effects model fits.

The peak velocity was mainly affected by the position of the object, but, notably, also by the available sensory inputs (Figure 6). The grasping movements in the unisensory conditions $(\mathrm{H}=948 \mathrm{~mm} / \mathrm{s}, 95 \%$ HDI $=906,990 ; \mathrm{V}=936 \mathrm{~mm} / \mathrm{s}, 95 \% \mathrm{HDI}=900,973)$ reached a lower peak velocity than in the multisensory condition $(\mathrm{VH}=971 \mathrm{~mm} / \mathrm{s}, 95 \% \mathrm{HDI}=948,1018)$, as can be also seen in Figure 7a. The comparisons between conditions (Figure $7 \mathrm{~d}$ ) showed that the peak velocity in $\mathrm{V}$ was credibly lower than in $\mathrm{VH}(\mathrm{V}-\mathrm{VH}=-47 \mathrm{~mm} / \mathrm{s}, 95 \% \mathrm{HDI}=-79,-16)$. No difference in terms of peak velocity was found between $\mathrm{H}$ and $\mathrm{V}(\mathrm{H}-\mathrm{V}=12 \mathrm{~mm} / \mathrm{s}, 95 \% \mathrm{HDI}=-33,55)$. Although the credible density distribution of 
the comparison between $\mathrm{H}$ and $\mathrm{VH}$ partially overlapped with zero $(\mathrm{H}-\mathrm{VH}=-35 \mathrm{~mm} / \mathrm{s}, 95 \% \mathrm{HDI}=-73$, 4), the bulk of the distribution was clearly negative. This pattern of results were very consistent across participants (Figure 8a, b, and c) and suggests that visual and haptic inputs are successfully integrated to speed-up multisensory grasping movements.
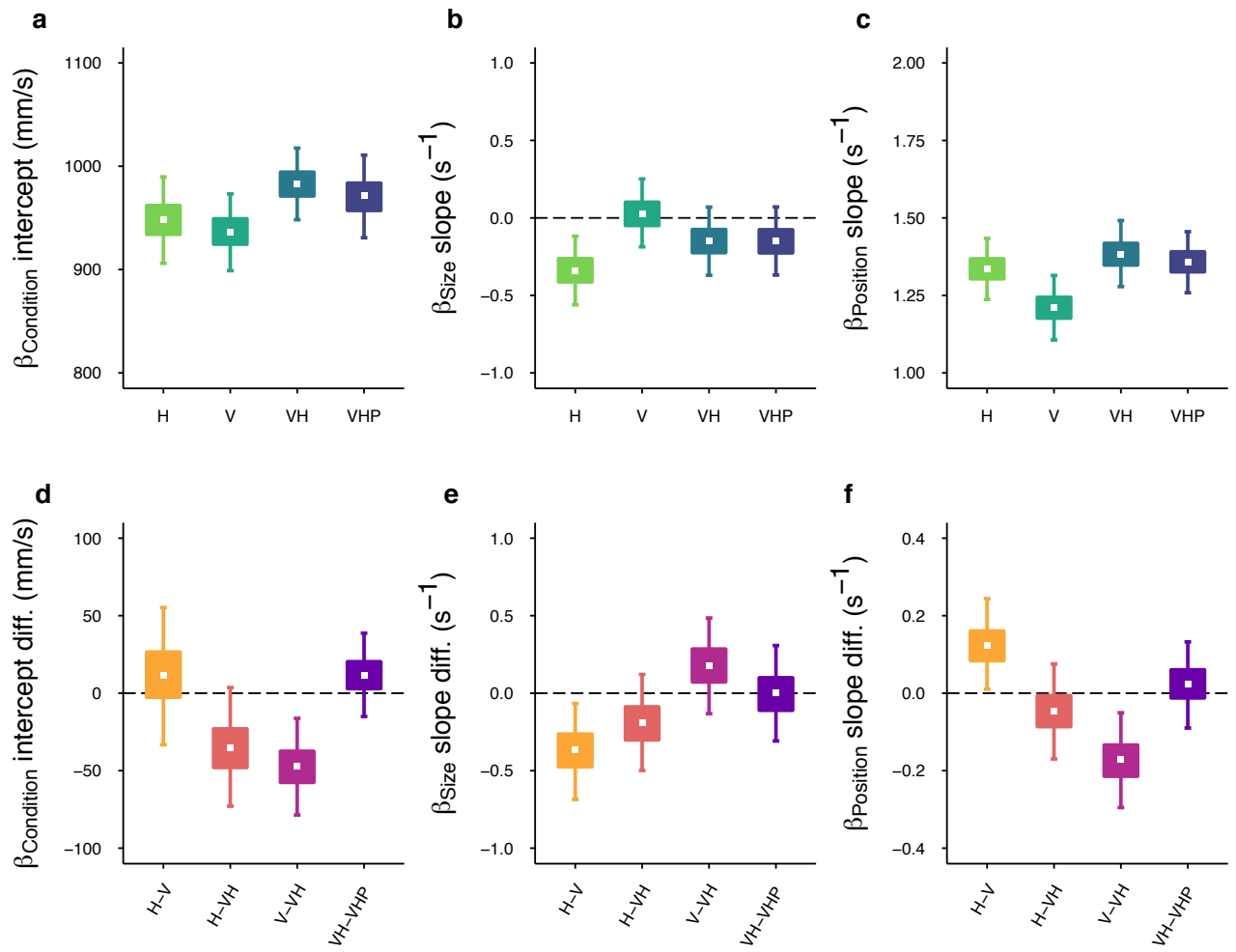

Figure 7: Peak velocity results. Top row: Posterior beta weights of the Bayesian linear mixed-effects regression model for the predictors Condition (a), Size (b) and Position (c). Bottom row: Credible difference distributions between conditions for the predictors Condition (d), Size (e) and Position (f). White dots represent the median, the boxes represent the $50 \%$ HDIs, and the areas between whiskers represent the $95 \%$ HDIs of the distributions.

The peak velocity was insensitive to changes in object size in $\mathrm{V}$ and $\mathrm{VH}$ conditions, as can be gathered from the slopes in Figure 7b, which were relatively flat both in $\mathrm{V}\left(\mathrm{V}=0.02 \mathrm{~s}^{-1}, 95 \% \mathrm{HDI}=-0.18,0.25\right)$ and in $\mathrm{VH}\left(\mathrm{VH}=-0.14 \mathrm{~s}^{-1}, 95 \% \mathrm{HDI}=-0.37,0.06\right)$. Instead, in $\mathrm{H}$, the peak velocity credibly decreased as a function of object size $\left(\mathrm{H}=-0.34 \mathrm{~s}^{-1}, 95 \% \mathrm{HDI}=-0.56,-0.12\right)$. This decrease might be due to a compensatory strategy specific to haptically-guided grasping wherein participants minimize the risk of colliding with larger objects by decreasing movement velocity instead of increasing the grip aperture. This might be also taken as a symptom of worse sensitivity to size in $\mathrm{H}$ than in $\mathrm{V}$. The comparisons between conditions (Figure 7e) showed that the slopes were credibly different only for the comparison between 
$\mathrm{H}$ and $\mathrm{V}\left(\mathrm{H}-\mathrm{V}=-0.36 \mathrm{~s}^{-1}, 95 \% \mathrm{HDI}=-0.68,-0.06\right)$. There was no evidence of any other difference $\left(\mathrm{H}-\mathrm{VH}=-0.19 \mathrm{~s}^{-1}, 95 \% \mathrm{HDI}=-0.49,0.12 ; \mathrm{V}-\mathrm{VH}=0.17 \mathrm{~s}^{-1}, 95 \% \mathrm{HDI}=-0.13,0.48\right)$.
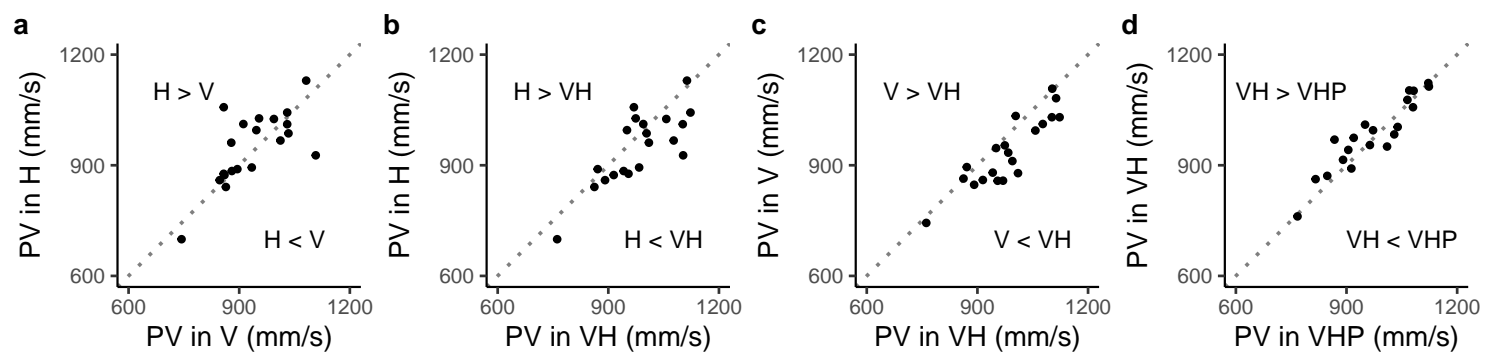

Figure 8: Scatterplots of paired observations. Each point represents the average peak velocity (PV) of a single participant for a pair of conditions: (a) V and $\mathrm{H}$, (b) VH and $\mathrm{H}$, (c) VH and V, (d) VHP and VH. The diagonal reference line of no effect has slope 1 and intercept 0 . Points above the diagonal line indicate that the peak velocity of the condition represented on the ordinate axis is larger than the peak velocity represented on the abscissa.

The object position credibly influenced peak velocity in all sensory conditions (Figure 7c). However, when haptic cues were available, either alone or in combination with vision, the increases in peak velocity as a function of object position were greater than when movements were under visual guidance only $(\mathrm{H}$ $=1.33 \mathrm{~s}^{-1}, 95 \% \mathrm{HDI}=1.23,1.43 ; \mathrm{V}=1.21 \mathrm{~s}^{-1}, 95 \% \mathrm{HDI}=1.10 \mathrm{~s}^{-1}, 1.31 ; \mathrm{VH}=1.38,95 \% \mathrm{HDI}=$ $1.27,1.49)$. This result was confirmed by the comparisons between conditions (Figure $7 f$ ). Whereas the peak velocity increases in $\mathrm{H}$ and $\mathrm{VH}$ were credibly different from those found in $\mathrm{V}\left(\mathrm{H}-\mathrm{V}=0.12 \mathrm{~s}^{-1}, 95 \%\right.$ $\left.\mathrm{HDI}=0.01,0.24 ; \mathrm{V}-\mathrm{VH}=-0.16 \mathrm{~s}^{-1}, 95 \% \mathrm{HDI}=-0.29,-0.05\right)$, no difference was observed between $\mathrm{H}$ and $\mathrm{VH}\left(\mathrm{H}-\mathrm{VH}=-0.04 \mathrm{~s}^{-1}, 95 \% \mathrm{HDI}=-0.16,0.07\right)$. These findings provide a first piece of evidence that multisensory integration in grasping relies on the haptic estimates of egocentric object distance more than on the haptic estimates of object size. The comparison between the VH and VHP conditions will further clarify the specific role of the individual haptic cues.

\subsection{Visuo-haptic grasping with only the haptic position cue}

If the haptic size cue is crucial to achieve faster movements with smaller grip apertures in multisensory grasping, then the absence of this cue should deteriorate grasping performance in VHP (Figure 1, left and middle panels) as predicted by the full integration and the size integration models. If, on the other hand, only the haptic position cue is sufficient to boost performance in multisensory grasping, the position integration model predicts that the grip apertures in VHP should be as small as in VH and movements 
should be as fast (Figure 1, right panel). Indeed, we found that grasping performance in VH and VHP was essentially identical (Figure 3 and 6).

The average peak grip aperture in VHP $(\mathrm{VHP}=80.3 \mathrm{~mm}, 95 \% \mathrm{HDI}=77.4,83.3)$ was the same as in $\mathrm{VH}$ (Figure 4d, $\mathrm{VH}-\mathrm{VHP}=0.9 \mathrm{~mm}, 95 \% \mathrm{HDI}=-1.1,2.9)$ and the peak grip aperture in VHP increased as a function of object size at the same rate as in $\mathrm{VH}(\mathrm{VHP}=0.73,95 \% \mathrm{HDI}=0.70,0.76)$, even when the haptic cue about object size was lacking (Figure 4e, VH-VHP $=0.03,95 \%$ HDI $=-0.01$, 0.06). With regards to object position, the peak grip aperture in VHP increased with distance $(0.008$, $95 \% \mathrm{HDI}=-0.001,0.001)$ as much as in VH (Figure 4f, VH-VHP $=0.002,95 \%$ HDI $=-0.006,0.01)$. Similarly, the average peak velocity in VHP $(971 \mathrm{~mm} / \mathrm{s}, 95 \%$ HDI $=931,1011)$ was the same as the one we observed in VH (Figure $7 \mathrm{~d}, \mathrm{VH}-\mathrm{VHP}=12 \mathrm{~mm} / \mathrm{s}, 95 \% \mathrm{HDI}=-15,39$ ) and the peak velocity in VHP (VHP $=-0.14 \mathrm{~s}^{-1}, 95 \%$ HDI $\left.=-0.36,0.07\right)$ was as insensitive to changes in object size as in VH (Figure 7e, VH-VHP $\left.=-0.0003 \mathrm{~s}^{-1}, 95 \% \mathrm{HDI}=-0.30,0.30\right)$. Finally, in terms of object position, the peak velocity scaling to distance did not differ between VH and VHP (VHP $=1.35 \mathrm{~s}^{-1}, 95 \%$ HDI $=$ 1.25, 1.45; VH-VHP $\left.=0.02 \mathrm{~s}^{-1}, 95 \% \mathrm{HDI}=-0.09,0.13\right)$. The similarities between $\mathrm{VH}$ and VHP in average peak grip aperture and average peak velocity can be clearly seen also at the per-participant level (Figures $5 \mathrm{~d}$ and $8 \mathrm{~d}$ ).
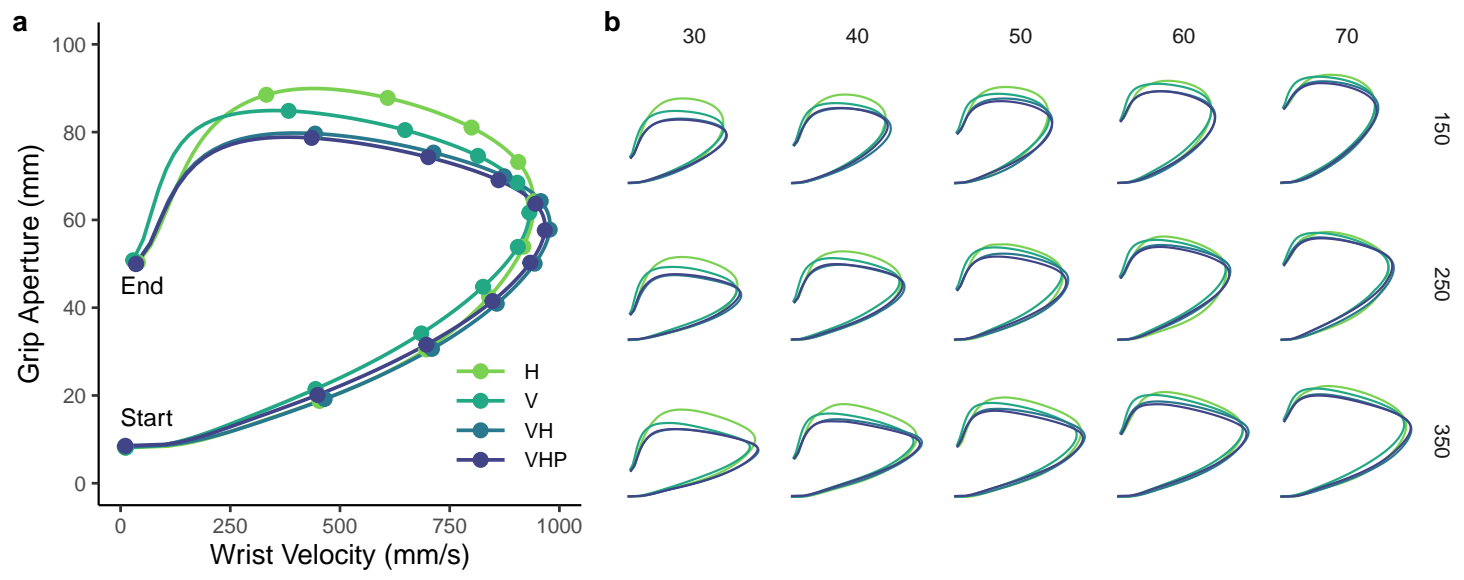

Figure 9: Wrist velocity and grip aperture from start to end of the movement. (a) H, V, VH and VHP conditions are represented by the lines obtained by resampling each movement trajectory in 201 steps evenly spaced along the three-dimensional path and by then averaging the wrist velocity and the grip aperture over all participants, sizes and positions, for each step of the movement trajectory. Points on the lines divide 10\% segments equally spaced along the movement trajectory. (b) Same as in (a), but each combination of size and position is represented separately. 
The comparable performance in VH and VHP conditions was also visible by tracking the evolution of the grip aperture and wrist velocity along the whole movement (Figure 9a). While the paths of the VH and VHP conditions in wrist velocity-grip aperture space clearly differ from the $\mathrm{V}$ and $\mathrm{H}$ conditions, they are essentially indistinguishable from each other for all the combinations of object size and position (Figure 9b).

Taken together, these results suggest that the haptic position cue, and not haptic size, is required to improve multisensory grasping movements. Thus, faster movements with smaller grip apertures are achieved by integrating visual and haptic position cues that are then jointly used to better scale visual size, consistent with the predictions of the position integration model.

\section{Discussion}

The study presented here was designed to investigate the role of visual and haptic cues in visuo-haptic multisensory grasping. The results show that the availability of both vision and haptics produced faster reach-to-grasp movements with considerably narrower grip apertures than in the unisensory conditions (vision-only or haptics-only) extending our previous findings (Camponogara \& Volcic, 2019a, 2019b) over a wide range of object sizes and object positions. Critically, when full vision was coupled with the haptic position cue only (VHP), grasping movements were indistinguishable from those found in the full visuo-haptic condition $(\mathrm{VH})$ in which also the haptic size cue was available.

Because both the full integration and the size integration models rely on the availability of the haptic size cue, its absence should have degraded grasping performance. Our results show that this was evidently not the case, and clearly support the position integration model. Therefore, we must conclude that the main contribution of the haptic modality is related to the estimation of the object position in space. The integration of this haptic position estimate with the available visual information is thus sufficient to enhance grasping performance to the same level as when also the haptic size cue is accessible. The use of haptics in supporting the visual estimation of the object position rather than its size might be related to the intrinsic positional function of the proprioceptive receptors, whose inputs provide a continuous flow of information about the extension of the limbs (Proske \& Gandevia, 2012). Our findings thus strengthen the idea that non-visual inputs from the hand holding the object actively support vision in estimating object properties (Battaglia et al., 2010; Carey \& Allan, 1996; Chen et al., 2018; Sperandio et al., 2013). 
The fact that the haptic size cue might only play a limited role in multisensory grasping might, at first glance, seem surprising. After all, the purpose of multisensory processing is to augment the information contributed by the single modalities (Ernst \& Bülthoff, 2004). However, a subordinate role of the haptic size cue could be seen as a virtue rather than a problem, because integrating this specific cue would be in many cases detrimental. Common objects have irregular shapes and, thus, the size perceived by the hand holding the object would rarely match the size of the part of the object that is the target of the grasping action (e.g., reaching with one hand for the cap of a bottle we hold in the other hand). Moreover, actions towards handheld objects do not necessarily end by grasping them (e.g., tapping on a smartphone). In these cases, the haptic position cue can still support vision in movement control, whereas the haptic size cue is essentially irrelevant. By relegating haptic size cues to a secondary role, the sensorimotor system could actually achieve greater robustness to variations in objects shape and action goals.

An additional point that is worth discussing concerns the role of multisensory grasping in learning the mapping between the visual and the motor systems. Infants start to develop the ability to reach and grasp objects at between four and six months of age before learning to perform grasping movements that resemble those of adults at the age of 10-12 months (Gonzalez \& Sacrey, 2018; Karl \& Whishaw, 2014). Importantly, their inability to produce successful grasps is not due to an immature motor system (Wallace \& Whishaw, 2003), to low visual acuity (Banks \& Salapatek, 1978) or to undeveloped stereovision (Braddick et al., 1980; Held, Birch, \& Gwiazda, 1980), but rather to an unformed mapping between visual inputs and motor plans. This visuomotor mapping is thought to be achieved through an embodied process that requires infants to rely on proprioceptive and, more generally, haptic inputs from their reaching hand (Corbetta, Thurman, Wiener, Guan, \& Williams, 2014; Corbetta, Wiener, Thurman, \& McMahon, 2018; Thomas, Karl, \& Whishaw, 2015). Our findings suggest that valuable haptic spatial cues provided by the hand holding the object could also assist the visuomotor learning process by promoting the development of precise visually controlled reach and grasp movements. In fact, anyone who has witnessed the motor development of a newborn must have observed that they can easily direct a grasping movement toward a handheld object long before they can grasp a distal object they can only see.

It is also important to note that the plasticity of the mapping between visual and motor systems does not end at the onset of adulthood. The visual feedback about the ongoing movement (Bozzacchi, Brenner, Smeets, Volcic, \& Domini, 2018; Connolly \& Goodale, 1999; Rand, Lemay, Squire, Shimansky, \& Stelmach, 2007; Schenk, Mair, \& Zihl, 2004; Schettino, Adamovich, \& Poizner, 2003; Volcic \& Domini, 2016; Winges, Weber, \& Santello, 2003), the terminal haptic feedback obtained by the grasping hand (Bingham, 
Coats, \& Mon-Williams, 2007; Bozzacchi, Volcic, \& Domini, 2014; Coats, Bingham, \& Mon-Williams, 2008; Mon-Williams \& Bingham, 2007; Weigelt \& Bock, 2007, 2010), and the sensory prediction errors from past trials (Tang, Whitwell, \& a. Goodale, 2015; Volcic \& Domini, 2018; Whitwell \& Goodale, 2009) have all been recognized to be important factors in calibrating visually guided grasping. We suggest that haptic spatial cues about the handheld object could presumably also play a relevant role in maintaining the correct visuomotor calibration over the lifespan or even aid the acquisition of visually guided skills in specific populations, such as children with dense bilateral congenital cataracts who recover vision years after birth (Chen et al., 2016; Held et al., 2011).

Even though this study was not designed to distinguish between different theories about grasping, it provides some important insights to be considered. First, according to the two-visual-systems hypothesis (Goodale, 2011), binocular vision provides estimates of distance and size that are accurate and reliable and is thus critical for grasping guidance. Indeed, switching from binocular to monocular vision has clear detrimental effects on grasping (Bradshaw et al., 2004; Melmoth \& Grant, 2006; Servos, Goodale, \& Jakobson, 1992). Thus, one might expect that providing a haptic position cue should compensate for the loss of visual distance cues in conditions of limited vision. This is, in fact, what has been found (Chen et al., 2018). Our finding that the haptic position cue reduces the grip aperture and increases movement velocity even in conditions of full binocular vision instead suggests that vision-for-action is less efficient than it is usually claimed. Second, our finding that holding the pole on which the object is placed (i.e., only haptic position cue available) leads to identical grasping performance as when both haptic size and position cues are available is not fully compatible with the theories about grasping that propose that either a single digit (Galea, Castiello, \& Dalwood, 2001; Haggard \& Wing, 1997; Melmoth \& Grant, 2012; Mon-Williams \& McIntosh, 2000; Wing \& Fraser, 1983) or both digits (Schot, Brenner, \& Smeets, 2017; Smeets \& Brenner, 1999; Smeets, van der Kooij, \& Brenner, 2019) are transported to specific positions on the object. In the VHP condition, the positions on the object toward which the fingers were moving (grasping points) did not coincide with the haptic positions felt by the fingers of the left hand. Integrating these visual and haptic position estimates should have thus led to a worse grasping performance than when these estimates were congruent (VH vs. VHP), which was however not the case. It is rather more plausible that multisensory grasping movements are planned and executed based on a visuo-haptic estimate of the object's centroid which is also used to better scale visual size. Alternatively, as suggested by one of the reviewers, the improvements observed in multisensory grasping movements might not be the result of a more reliable scaling of visual size, but rather the consequence of 
more successful sensory transformations that could facilitate the estimation of the grasping points on the object. Seeing the left hand holding the object and the haptic position cues provided by the same hand could assist the realignment of the grasping points that are initially available in different modality-specific coordinates (Kuling, Brenner, \& Smeets, 2016; Kuling, van der Graaff, Brenner, \& Smeets, 2017; Smeets, van den Dobbelsteen, de Grave, van Beers, \& Brenner, 2006). This view might also explain why the contribution of haptic cues might, in specific cases, not be beneficial (Brenner et al., 1997).

In summary, our study provides a new perspective on the role of haptics in sensorimotor control by highlighting how non-visual inputs influence vision during object manipulation. Whether it be reaching for the cap of a handheld bottle, or passing a glass from one hand to the other, humans rely on haptics to guide movements more than we realize. Often, haptics can even take a leading role when vision is driven away from the manipulated object (e.g., when looking at a glass while uncorking a bottle). Hence, haptics is a remarkably underestimated source of information we draw from to guide efficient reaching and grasping movements in everyday life.

\section{Acknowledgements}

We thank Prof. Jeroen Smeets for helpful comments and discussions.

\section{Data Availability}

Experimental data described in this manuscript are available on Open Science Framework following this link: https://osf.io/apyks/. The code for the data analysis is also available at the same link.

\section{CRediT author statement}

Ivan Camponogara: Conceptualization, Methodology, Software, Validation, Formal analysis, Investigation, Data Curation, Writing - Original Draft, Writing - Review \& Editing, Visualization. Robert Volcic: Conceptualization, Methodology, Software, Validation, Formal analysis, Data Curation, Resources, Writing - Review \& Editing, Visualization, Supervision. 
bioRxiv preprint doi: https://doi.org/10.1101/2020.05.12.090647; this version posted November 12, 2020. The copyright holder for this

preprint (which was not certified by peer review) is the author/funder, who has granted bioRxiv a license to display the preprint in

perpetuity. It is made available under aCC-BY-NC-ND 4.0 International license.

\section{Additional information}

Competing interests The authors declare no competing interests.

Correspondence and requests for materials should be addressed to I.C. 


\section{References}

Banks, M. S., \& Salapatek, P. (1978). Acuity and contrast sensitivity in 1-, 2-, and 3-month-old human infants. Investigative Ophthalmology \& Visual Science, 17, 361-365.

Battaglia, P. W., Di Luca, M., Ernst, M. O., Schrater, P. R., Machulla, T., \& Kersten, D. (2010). Within- and cross-modal distance information disambiguate visual size-change perception. PLoS Computational Biology, 6(3), e1000697. doi: 10.1371/journal.pcbi.1000697

Berryman, L. J., Yau, J. M., \& Hsiao, S. S. (2006). Representation of object size in the somatosensory system. Journal of Neurophysiology, 96(1), 27-39. doi: 10.1152/jn.01190.2005

Bingham, G. P., Coats, R., \& Mon-Williams, M. (2007). Natural prehension in trials without haptic feedback but only when calibration is allowed. Neuropsychologia , 45(2), 288-294. doi: 10.1016/j.neuropsychologia.2006.07.011

Bozzacchi, C., Brenner, E., Smeets, J. B., Volcic, R., \& Domini, F. (2018). How removing visual information affects grasping movements. Experimental Brain Research, 236(4), 985-995. doi: 10.1007/s00221-018-5186-6

Bozzacchi, C., Volcic, R., \& Domini, F. (2014). Effect of visual and haptic feedback on grasping movements. Journal of Neurophysiology, 112, 3189-3196. doi: 10.1152/jn.00439.2014

Braddick, O., Atkinson, J., Julesz, B., Kropfl, W., Bodis-Wollner, I., \& Raab, E. (1980). Cortical binocularity in infants. Nature, 288(5789), 363-365. doi: 10.1038/288363a0

Bradshaw, M. F., Elliott, K. M., Watt, S. J., Hibbard, P. B., Davies, I. R., \& Simpson, P. J. (2004). Binocular cues and the control of prehension. Spatial Vision, 17(1-2), 95-110. doi: $10.1163 / 156856804322778288$

Brenner, E., \& van Damme, W. J. (1999). Perceived distance, shape and size. Vision Research, 39(5), 975-986. doi: 10.1016/S0042-6989(98)00162-X

Brenner, E., van Damme, W. J., \& Smeets, J. B. J. (1997). Holding an object one is looking at: Kinesthetic information on the object's distance does not improve visual judgments of its size. Perception \& Psychophysics, 59(7), 1153-1159. doi: 10.3758/BF03205528 
Bürkner, P.-C. (2017). brms: An R package for Bayesian Generalized Linear Mixed Models using Stan. Journal of Statistical Software, 80(1), 1-28. doi: 10.18637/jss.v080.i01

Camponogara, I., \& Volcic, R. (2019a). Grasping adjustments to haptic, visual, and visuo-haptic object perturbations are contingent on the sensory modality. Journal of Neurophysiology, 122(6), 2614-2620. doi: 10.1152/jn.00452.2019

Camponogara, I., \& Volcic, R. (2019b). Grasping movements toward seen and handheld objects. Scientific Reports, 9(1), 3665. doi: 10.1038/s41598-018-38277-w

Carey, D. P., \& Allan, K. (1996). A motor signal and "visual" size perception. Experimental Brain Research, 110(3), 482-486. doi: 10.1007/BF00229148

Carpenter, B., Gelman, A., Hoffman, M. D., Lee, D., Goodrich, B., Betancourt, M., ... Riddell, A. (2017). Stan: A Probabilistic Programming Language. Journal of Statistical Software, 76 (1), 1-32. doi: $10.18637 /$ jss.v076.i01

Castiello, U. (2005). The neuroscience of grasping. Nature Reviews Neuroscience, 6(9), 726-736. doi: $10.1038 / \mathrm{nrn} 1744$

Chen, J., Sperandio, I., \& Goodale, M. A. (2018). Proprioceptive distance cues restore perfect size constancy in grasping, but not perception, when vision is limited. Current Biology, 28(6), 927-932. doi: 10.1016/j.cub.2018.01.076

Chen, J., Wu, E.-D., Chen, X., Zhu, L.-H., Li, X., Thorn, F., ... Qu, J. (2016). Rapid integration of tactile and visual information by a newly sighted child. Current Biology, 26(8), 1069-1074. doi: 10.1016/j.cub.2016.02.065

Coats, R., Bingham, G. P., \& Mon-Williams, M. (2008). Calibrating grasp size and reach distance: Interactions reveal integral organization of reaching-to-grasp movements. Experimental Brain Research, 189(2), 211-220. doi: 10.1007/s00221-008-1418-5

Connolly, J. D., \& Goodale, M. A. (1999). The role of visual feedback of hand position in the control of manual prehension. Experimental Brain Research, 125(3), 281-286. doi: $10.1007 / \mathrm{s} 002210050684$

Corbetta, D., Thurman, S. L., Wiener, R. F., Guan, Y., \& Williams, J. L. (2014). Mapping the feel of the arm with the sight of the object: On the embodied origins of infant reaching. 
Frontiers in Psychology, 5. doi: 10.3389/fpsyg.2014.00576

Corbetta, D., Wiener, R. F., Thurman, S. L., \& McMahon, E. (2018). The embodied origins of infant reaching: Implications for the emergence of eye-hand coordination. Kinesiology Review, 7(1), 10-17. doi: 10.1123/kr.2017-0052

Derzsi, Z., \& Volcic, R. (2018). MOTOM toolbox: MOtion Tracking via Optotrak and Matlab. Journal of Neuroscience Methods, 308, 129-134. doi: 10.1016/j.jneumeth.2018.07.007

Durlach, N. I., a Delhorne, L., Wong, A., Ko, W. Y., Rabinowitz, W. M., \& Hollerbach, J. (1989). Manual discrimination and identification of length by the finger-span method. Perception \& Psychophysics, 46(1), 29-38. doi: 10.3758/BF03208071

Epstein, W., Park, J., \& Casey, A. (1961). The current status of the size-distance hypotheses. Psychological Bulletin, 58(6), 491-514. doi: 10.1037/h0042260

Ernst, M. O., \& Banks, M. S. (2002). Humans integrate visual and haptic information in a statistically optimal fashion. Nature, 415(6870), 429-433. doi: 10.1038/415429a

Ernst, M. O., \& Bülthoff, H. H. (2004). Merging the senses into a robust percept. Trends in Cognitive Sciences, 8(4), 162-169. doi: 10.1016/j.tics.2004.02.002

Galea, M. P., Castiello, U., \& Dalwood, N. (2001). Thumb invariance during prehension movement: Effects of object orientation. Neuroreport, 12(10), 2185-2187.

Gaydos, H. F. (1958). Sensitivity in the judgment of size by finger-span. The American Journal of Psychology, 71(3), 557-562. doi: 10.2307/1420251

Gepshtein, S., \& Banks, M. S. (2003). Viewing geometry determines how vision and haptics combine in size perception. Current Biology, 13(6), 483-488. doi: 10.1016/S09609822(03)00133-7

Gepshtein, S., Burge, J., Ernst, M. O., \& Banks, M. S. (2005). The combination of vision and touch depends on spatial proximity. Journal of Vision, 5(11), 1013-1023. doi: $10.1167 / 5.11 .7$

Gonzalez, C. L., \& Sacrey, L.-A. R. (2018). The development of the motor system. In R. Gibb \& B. Kolb (Eds.), The neurobiology of brain and behavioral development (pp. 235-256). Academic Press. doi: 10.1016/B978-0-12-804036-2.00009-1 
Goodale, M. A. (2011). Transforming vision into action. Vision Research, 51(13), 1567-1587. doi: $10.1016 /$ j.visres.2010.07.027

Haggard, P., \& Wing, A. (1997). On the hand transport component of prehensile movements. Journal of Motor Behavior, 29(3), 282-287. doi: 10.1080/00222899709600842

Held, R., Birch, E., \& Gwiazda, J. (1980). Stereoacuity of human infants. Proceedings of the National Academy of Sciences, 77(9), 5572-5574. doi: 10.1073/pnas.77.9.5572

Held, R., Ostrovsky, Y., de Gelder, B., Gandhi, T., Ganesh, S., Mathur, U., \& Sinha, P. (2011). The newly sighted fail to match seen with felt. Nature Neuroscience, 14(5), 551-553. doi: $10.1038 / \mathrm{nn} .2795$

Janssen, P., \& Scherberger, H. (2015). Visual guidance in control of grasping. Annual Review of Neuroscience, 38(1), 69-86. doi: 10.1146/annurev-neuro-071714-034028

Jeannerod, M. (1981). Intersegmental coordination during reaching at natural visual objects. In A. Berthoz, J. B. Long, \& A. D. Baddeley (Eds.), Attention and performance IX: Proceedings of the 9th International Symposium on Attention and Performance, Cambridge, July 13-18, 1980 (pp. 153-169). Erlbaum.

Jeannerod, M., Arbib, M. A., Rizzolatti, G., \& Sakata, H. (1995). Grasping objects: The cortical mechanisms of visuomotor transformation. Trends in Neurosciences, 18(7), 314-320. doi: 10.1016/0166-2236(95)93921-J

Karl, J. M., \& Whishaw, I. Q. (2014). Haptic grasping configurations in early infancy reveal different developmental profiles for visual guidance of the Reach versus the Grasp. Experimental Brain Research, 3301-3316. doi: 10.1007/s00221-014-4013-y

Kuling, I. A., Brenner, E., \& Smeets, J. B. (2016). Errors in visuo-haptic and haptic-haptic location matching are stable over long periods of time. Acta Psychologica, 166, 31-36. doi: 10.1016/j.actpsy.2016.03.011

Kuling, I. A., van der Graaff, M. C. W., Brenner, E., \& Smeets, J. B. J. (2017). Matching locations is not just matching sensory representations. Experimental Brain Research, 235(2), 533-545. doi: 10.1007/s00221-016-4815-1

Langfeld, H. S. (1917). The differential spatial limen for finger span. Journal of Experimental 
Psychology, 2(6), 416-430. doi: 10.1037/h0070182

Melmoth, D. R., \& Grant, S. (2006). Advantages of binocular vision for the control of reaching and grasping. Experimental Brain Research, 171(3), 371-388. doi: 10.1007/s00221-005$0273-\mathrm{x}$

Melmoth, D. R., \& Grant, S. (2012). Getting a grip: Different actions and visual guidance of the thumb and finger in precision grasping. Experimental Brain Research, 222(3), 265-276. doi: $10.1007 / \mathrm{s} 00221-012-3214-5$

Mon-Williams, M., \& Bingham, G. P. (2007). Calibrating reach distance to visual targets. Journal of Experimental Psychology: Human Perception and Performance, 33(3), 645656. doi: $10.1037 / 0096-1523.33 .3 .645$

Mon-Williams, M., \& McIntosh, R. D. (2000). A test between two hypotheses and a possible third way for the control of prehension. Experimental Brain Research, 134(2), 268-273. doi: $10.1007 / \mathrm{s} 002210000479$

Nicolini, C., Fantoni, C., Mancuso, G., Volcic, R., \& Domini, F. (2014). A framework for the study of vision in active observers. In B. E. Rogowitz, T. N. Pappas, \& H. de Ridder (Eds.), Proceedings of SPIE, Human Vision and Electronic Imaging XIX (Vol. 9014, p. 901414). doi: $10.1117 / 12.2045459$

Pettypiece, C. E., Culham, J. C., \& Goodale, M. A. (2009). Differential effects of delay upon visually and haptically guided grasping and perceptual judgments. Experimental Brain Research, 195(3), 473-479. doi: 10.1007/s00221-009-1807-4

Pettypiece, C. E., Goodale, M. A., \& Culham, J. C. (2010). Integration of haptic and visual size cues in perception and action revealed through cross-modal conflict. Experimental Brain Research, 201(4), 863-873. doi: 10.1007/s00221-009-2101-1

Proske, U., \& Gandevia, S. C. (2012). The proprioceptive senses: Their roles in signaling body shape, body position and movement, and muscle force. Physiological Reviews, 92(4), 1651-1697. doi: 10.1152/physrev.00048.2011

R Core Team. (2019). R: A language and environment for statistical computing [manual]. Retrieved from https://www.R-project.org/ 
Rand, M., Lemay, M., Squire, L., Shimansky, Y., \& Stelmach, G. (2007). Role of vision in aperture closure control during reach-to-grasp movements. Experimental Brain Research, $181(3)$, 447-460. doi: 10.1007/s00221-007-0945-9

Schenk, T., Mair, B., \& Zihl, J. (2004). The use of visual feedback and on-line target information in catching and grasping. Experimental Brain Research, 154, 85-96. doi: 10.1007/s00221003-1642-y

Schettino, L., Adamovich, S. V., \& Poizner, H. (2003). Effects of object shape and visual feedback on hand configuration during grasping. Experimental Brain Research, 151(2), 158-166. doi: 10.1007/s00221-003-1435-3

Schot, W. D., Brenner, E., \& Smeets, J. B. (2017). Unusual prism adaptation reveals how grasping is controlled. eLife, 6. doi: 10.7554/eLife.21440

Schot, W. D., Brenner, E., \& Smeets, J. B. J. (2010). Robust movement segmentation by combining multiple sources of information. Journal of Neuroscience Methods, 187(2), 147-155. doi: 10.1016/j.jneumeth.2010.01.004

Servos, P., Goodale, M. A., \& Jakobson, L. S. (1992). The role of binocular vision in prehension: A kinematic analysis. Vision Research, 32(8), 1513-1521. doi: 10.1016/00426989(92)90207-Y

Smeets, J. B. J., \& Brenner, E. (1999). A new view on grasping. Motor Control, 3(3), 237-271. doi: $10.1123 / \operatorname{mcj} .3 .3 .237$

Smeets, J. B. J., van den Dobbelsteen, J. J., de Grave, D. D., van Beers, R. J., \& Brenner, E. (2006). Sensory integration does not lead to sensory calibration. Proceedings of the National Academy of Sciences, 103(49), 18781-18786. doi: 10.1073/pnas.0607687103

Smeets, J. B. J., van der Kooij, K., \& Brenner, E. (2019). A review of grasping as the movements of digits in space. Journal of Neurophysiology, 122(4), 1578-1597. doi: $10.1152 /$ jn. 00123.2019

Sperandio, I., Kaderali, S., Chouinard, P. A., Frey, J., \& Goodale, M. A. (2013). Perceived size change induced by nonvisual signals in darkness: The relative contribution of vergence and proprioception. Journal of neuroscience, 33(43), 16915-16923. doi: 


\subsection{3/JNEUROSCI.0977-13.2013}

Tang, R., Whitwell, R. L., \& a. Goodale, M. (2015). The influence of visual feedback from the recent past on the programming of grip aperture is grasp-specific, shared between hands, and mediated by sensorimotor memory not task set. Cognition, 138, 49-63. doi: 10.1016/j.cognition.2015.01.012

Thomas, B. L., Karl, J. M., \& Whishaw, I. Q. (2015). Independent development of the Reach and the Grasp in spontaneous self-touching by human infants in the first 6 months. Frontiers in Psychology, 5. doi: 10.3389/fpsyg.2014.01526

van Damme, W., \& Brenner, E. (1997). The distance used for scaling disparities is the same as the one used for scaling retinal size. Vision Research, 37(6), 757-764. doi: 10.1016/S00426989(96)00213-1

Volcic, R., \& Domini, F. (2016). On-line visual control of grasping movements. Experimental Brain Research, 234(8), 2165-2177. doi: 10.1007/s00221-016-4620-x

Volcic, R., \& Domini, F. (2018). The endless visuomotor calibration of reach-to-grasp actions. Scientific Reports, 8(1), 14803. doi: 10.1038/s41598-018-33009-6

Volcic, R., Fantoni, C., Caudek, C., Assad, J. A., \& Domini, F. (2013). Visuomotor adaptation changes stereoscopic depth perception and tactile discrimination. Journal of Neuroscience, 33(43), 17081-8. doi: 10.1523/JNEUROSCI.2936-13.2013

Wallace, P. S., \& Whishaw, I. Q. (2003). Independent digit movements and precision grip patterns in 1-5-month-old human infants: Hand-babbling, including vacuous then selfdirected hand and digit movements, precedes targeted reaching. Neuropsychologia, 41(14), 1912-1918. doi: 10.1016/S0028-3932(03)00128-3

Weigelt, C., \& Bock, O. (2007). Adaptation of grasping responses to distorted object size and orientation. Experimental Brain Research, 181(1), 139-146. doi: 10.1007/s00221-0070911-6

Weigelt, C., \& Bock, O. (2010). Adaptation of the precision grip orientation to a visual-haptic mismatch. Experimental Brain Research, 201(4), 621-630. doi: 10.1007/s00221-009-2076y 
Whitwell, R. L., \& Goodale, M. A. (2009). Updating the programming of a precision grip is a function of recent history of available feedback. Experimental Brain Research, 194(4), 619-629. doi: 10.1007/s00221-009-1737-1

Wing, A. M., \& Fraser, C. (1983). The contribution of the thumb to reaching movements. The Quarterly Journal of Experimental Psychology A, 35, 297-309. doi: $10.1080 / 14640748308402135$

Winges, S., Weber, D., \& Santello, M. (2003). The role of vision on hand preshaping during reach to grasp. Experimental Brain Research, 152(4), 489-498. doi: 10.1007/s00221-003-1571-9 